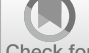

Check for

updates

Cite as

Nano-Micro Lett.

(2020) 12:41

Received: 8 November 2019

Accepted: 23 December 2019

Published online: 27 January 2020

(C) The Author(s) 2020

\section{Highly Dispersed RuOOH Nanoparticles on Silica Spheres: An Efficient Photothermal Catalyst for Selective Aerobic Oxidation of Benzyl Alcohol}

\author{
Qilin Wei ${ }^{1}$, Kiersten G. Guzman ${ }^{1}$, Xinyan Dai ${ }^{1}$, Nuwan H. Attanayake ${ }^{1}$, \\ Daniel R. Strongin ${ }^{1}$, Yugang $\operatorname{Sun}^{1} \bowtie$ \\ $\triangle$ Yugang Sun,ygsun@temple.edu \\ 1 Department of Chemistry, Temple University, 1901 North 13th Street, Philadelphia, PA 19122, USA
}

\title{
HIGHLIGHTS
}

- Ultrasmall RuOOH nanoparticles of 2-3 nm are loaded on submicron silica spheres and capable of activating molecular oxygen.

- Photothermal conversion efficiency of the supported RuOOH nanoparticles is nearly unity.

- Photothermal effect promotes selective oxidation of benzyl alcohol under the illumination of visible light.

\begin{abstract}
Photothermal catalysis represents a promising strategy to utilize the renewable energy source (e.g., solar energy) to drive chemical reactions more efficiently. Successful and efficient photothermal catalysis relies on the availability of ideal photothermal catalysts, which can provide both large areas of catalytically active surface and strong light absorption power simultaneously. Such duplex requirements of a photothermal catalyst exhibit opposing dependence on the size of the catalyst nanoparticles, i.e., smaller size is beneficial for achieving higher surface area and more active surface, whereas larger size favors the light absorption in the nanoparticles. In this article, we report the synthesis of ultrafine $\mathrm{RuOOH}$ nanoparticles with a size of 2-3 nm uniformly dispersed on the surfaces of silica $\left(\mathrm{SiO}_{x}\right)$ nanospheres of hundreds of nanometers in size

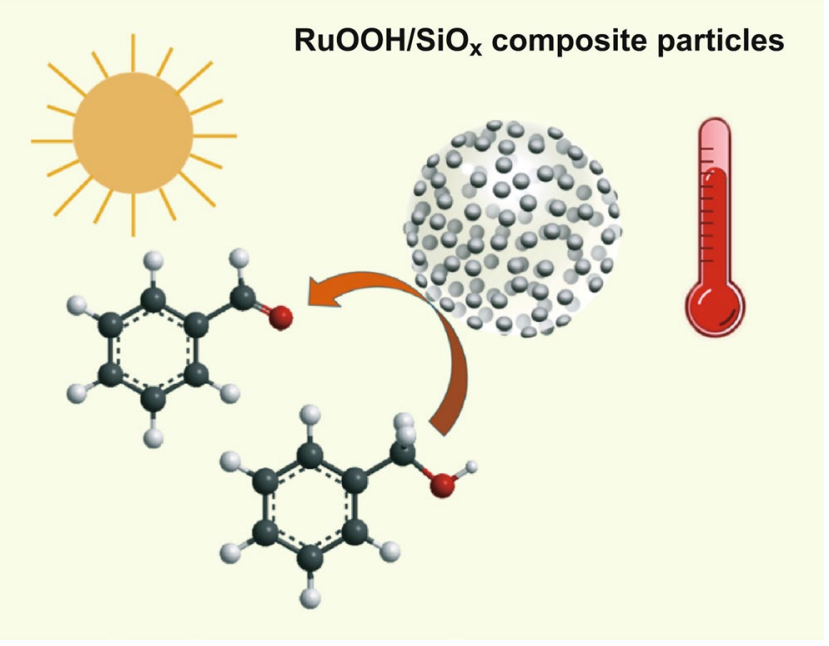
to tackle this challenge of forming an ideal photothermal catalyst.

The ultrasmall $\mathrm{RuOOH}$ nanoparticles exhibit a large surface area as well as the ability to activate adsorbed molecular oxygen. The $\mathrm{SiO}_{x}$ nanospheres exhibit strong surface light scattering resonances to enhance the light absorption power of the small RuOOH nanoparticles anchored on the $\mathrm{SiO}_{x}$ surface. Therefore, the $\mathrm{RuOOH} / \mathrm{SiO}_{x}$ composite particles represent a new class of efficient photothermal catalysts with a photothermal energy conversion efficiency of $92.5 \%$ for selective aerobic oxidation of benzyl alcohol to benzylaldehyde under ambient conditions.
\end{abstract}

KEYWORDS Ultrasmall RuOOH nanoparticles; Photothermal catalyst; Selective aerobic oxidation; Light antenna effect; Light scattering resonance 


\section{Introduction}

Selective aerobic oxidation of primary alcohols to corresponding aldehydes is of great interest in the chemical synthesis industry because of the environment-friendly and cost-effective features [1-4]. The high bond dissociation energy of $\alpha-\mathrm{C}-\mathrm{H}$ and weak oxidizing power of molecular oxygen $\left(\mathrm{O}_{2}\right)$ lead to challenges for successful reactions under mild ambient conditions [5-8]. The promising solution relies on the use of appropriate catalysts and elevated temperatures $\left(80-120{ }^{\circ} \mathrm{C}\right.$, if necessary) $[1,9]$. The general principle of designing a catalyst is to reduce the size of catalyst nanoparticles to maximize their surface area, simultaneously increasing their catalytic activity and lowering the usage of catalyst materials (in particular precious metals) $[10,11]$. In this work, we introduce highly dispersed RuOOH nanoparticles (NPs) with sizes of 2-3 nm that are synthesized through the simple hydrolysis of $\mathrm{Ru}(\mathrm{III})$ salt as a unique catalyst for selective aerobic oxidation of benzyl alcohol (BzOH) to benzylaldehyde (BzAD). The corresponding oxidation reaction highly depends on the partial pressure of $\mathrm{O}_{2}$, significantly different from the hydrated $\mathrm{RuO}_{2}$ and $\mathrm{Ru}(\mathrm{OH})_{3}$ nanoparticle (NP) catalysts reported in the literature, on which the oxidation reactions are independent of the partial pressure of $\mathrm{O}_{2}$ [12-15]. The change in reaction mechanism enables the aerobic oxidation of $\mathrm{BzOH}$ at mild temperatures and an additional strategy for tuning reaction rate by varying the partial pressure of $\mathrm{O}_{2}$.

The lowered reaction temperature makes it appropriate to use the more energy-efficient way, such as photothermal effect [16], to heat the reaction solution since the $\mathrm{RuOOH}$ NPs can absorb light of a broad spectral region, including visible light [17]. The extremely large surface-to-volume ratio of the ultrafine $\mathrm{RuOOH}$ catalyst NPs can significantly lower the usage of total $\mathrm{RuOOH}$ material compared to the reactions catalyzed with large-sized NPs. However, the small size of the RuOOH NPs with 2-3 nm in diameters limits their light absorption power. This challenge is tackled by loading the RuOOH catalyst NPs onto silica nanospheres $\left(\mathrm{SiO}_{x} \mathrm{NSs}\right)$ with an average diameter of $443 \mathrm{~nm}$, resulting in a significant increase in colloidal stability of the RuOOH NPs. The $\mathrm{SiO}_{x}$ NSs with the maximum geometric symmetry also represent a novel class of dielectric antennae that can produce enhanced electric fields near the particle surface upon light illumination due to the surface light scattering resonances [18]. Such an antenna effect benefits the light absorption efficiency of small nanoparticles loaded on the surfaces of the $\mathrm{SiO}_{x}$ NSs to promote light harvesting and the following energy conversion processes, such as hot-electron-driven chemical transformation [19-21] and photothermal-induced phenomena [22]. The $\mathrm{RuOOH} / \mathrm{SiO}_{x}$ composite particles behave as a new class of photothermal catalysts for selective aerobic oxidation of primary alcohols with an energy conversion efficiency of as high as $92.5 \%$.

\section{Materials and Methods}

\subsection{Materials Synthesis}

Synthesis of $\mathrm{SiO}_{x}$ NSs The synthesis relied on the controlled hydrolysis of tetraethyl orthosilicate (TEOS, 98\%, Sigma-Aldrich) and condensation of the hydrolyzates [18]. In a typical synthesis, $19.6 \mathrm{~mL}$ of ammonia hydroxide solution (28-30 wt\% in water, Fisher Scientific) was added to a mixture of $32 \mathrm{~mL}$ of deionized (DI) $\mathrm{H}_{2} \mathrm{O}$ and $260 \mathrm{~mL}$ of 190-proof ethanol. To this solution was then injected $17 \mathrm{~mL}$ of TEOS. The magnetic stirring at a rate of $600 \mathrm{rpm}$ (revolutions per min) and the room temperature were maintained throughout the entire synthesis for $2 \mathrm{~h}$. The resulting $\mathrm{SiO}_{x}$ NSs were collected by centrifugation and washed with ethanol to remove the unreacted species. The collected $\mathrm{SiO}_{x} \mathrm{NSs}$ were dried overnight in an oven set at $60{ }^{\circ} \mathrm{C}$.

Surface modification of the $\mathrm{SiO}_{x}$ NSs Dispersing $400 \mathrm{mg}$ of the dried $\mathrm{SiO}_{x}$ NSs in $200 \mathrm{~mL}$ of ethanol was carried out with the assistance of ultrasonication. The dispersion was heated up to $60{ }^{\circ} \mathrm{C}$ followed by a dropwise addition of $2 \mathrm{~mL}$ of (3-aminopropyl)triethoxysilane (APTES, 98\%, Acros Organics) at a rate of $1 \mathrm{~mL} \mathrm{~min}{ }^{-1}$. The dispersion was continuously incubated at $60{ }^{\circ} \mathrm{C}$ for $8 \mathrm{~h}$ while a magnetic stirring was maintained. This process linked APTES to the surfaces of the $\mathrm{SiO}_{x}$ NSs. The APTES-modified $\mathrm{SiO}_{x}$ NSs were then collected by centrifugation and washed with ethanol. The powder was dried in the air at ambient conditions.

Deposition of RuOOH NPs on the SiO ${ }_{x}$ NSs $100 \mathrm{mg}$ of APTES-modified $\mathrm{SiO}_{x}$ NSs was first dispersed in $50 \mathrm{~mL}$ 
of DI $\mathrm{H}_{2} \mathrm{O}$. To this dispersion was injected $1 \mathrm{~mL}$ of an aqueous solution containing $3.09 \mathrm{mg}(11.88 \mu \mathrm{mol})$ of ruthenium trichloride hydrate $\left(\mathrm{RuCl}_{3} \cdot x \mathrm{H}_{2} \mathrm{O}\right.$, Alfa Aesar). Hydrolysis of $\mathrm{RuCl}_{3}$ in water deposited $\mathrm{Ru}(\mathrm{III})$ hydrolyzate on the surfaces of the $\mathrm{SiO}_{x} \mathrm{NSs}$ that provided sufficient nucleation sites for forming nanoparticles. After $1 \mathrm{~h}$, the resulting composite particles were collected by centrifugation and washed with DI $\mathrm{H}_{2} \mathrm{O}$. Inductively coupled plasma atomic emission spectroscopy (ICP-OES) was used to analyze the loading of $\mathrm{Ru}(\mathrm{III})$ species on the $\mathrm{SiO}_{x} \mathrm{NSs}$, showing $1.1 \mathrm{wt} \%$ loading of $\mathrm{Ru}$ (III) that corresponded to $92 \%$ of the feeding precursor. The composite particles were then dried in an oven set at $60{ }^{\circ} \mathrm{C}$ for $1 \mathrm{~h}$, followed by mild thermal annealing in a tube furnace set at $150{ }^{\circ} \mathrm{C}$ for $1 \mathrm{~h}$. The thermal annealing was performed in the ambient air atmosphere. The resulting supported $\mathrm{RuOOH}$ NPs exhibit very small sizes in the range of 2-3 nm.

Direct synthesis of $\mathrm{RuOOH}$ powders An aqueous solution of APTES was prepared by adding $200 \mu \mathrm{L}$ of APTES to $10 \mathrm{~mL}$ of $\mathrm{DI} \mathrm{H}_{2} \mathrm{O}$ under vigorous magnetic stirring. To this solution was injected $20 \mathrm{~mL}$ of an aqueous solution containing $100 \mathrm{mg}$ of $\mathrm{RuCl}_{3} \cdot x \mathrm{H}_{2} \mathrm{O}$. After the reaction lasted $1 \mathrm{~h}$, the powder was collected by centrifugation and washed by DI $\mathrm{H}_{2} \mathrm{O}$ twice. The as-prepared freestanding $\mathrm{RuOOH}$ powder was dried in an oven and annealed in a furnace, as same as the $\mathrm{RuOOH} / \mathrm{SiO}_{x}$ composite powders. When the concentration of APTES was significantly increased to $3 \mathrm{~mL}$ and the mass of $\mathrm{RuCl}_{3} \cdot x \mathrm{H}_{2} \mathrm{O}$ was decreased to $30 \mathrm{mg}$, the basicity of the reaction solution enhanced to promote the rapid hydrolysis of $\mathrm{Ru}(\mathrm{III})$ and the high-concentration APTES also promoted its self-hydrolysis to form $\mathrm{SiO}_{x} \mathrm{NSs}$. As a result, the simultaneous hydrolysis of Ru(III) and APTES formed $\mathrm{RuOOH} / \mathrm{SiO}_{x}$ composite particles with agglomerated $\mathrm{RuOOH}$ NPs of large sizes.

\subsection{Characterizations}

Scanning electron microscopy (SEM) images were obtained using a field-emission microscope (FEI Quanta FEG 450) operated at an acceleration voltage of $10 \mathrm{kV}$ in high vacuum mode. Transmission electron microscopy (TEM) images were taken using the JEOL JEM-1400 microscope operated at $120 \mathrm{kV}$. X-ray photoelectron spectra (XPS) were recorded using a VG Scientific $100 \mathrm{~mm}$ hemispherical analyzer equipped with a Physical Electronics $\mathrm{Mg} \mathrm{K} \alpha \mathrm{X}$-ray source operating at $300 \mathrm{~W}$. Raman spectroscopy was analyzed with a Horiba Jobin-Yvon Labram HR800 Evolution confocal Raman spectrometer using a 532-nm laser. The Olympus MPlan N 100×microscope objective was used to focus the excitation laser down to a spot size of $\sim 1 \mu \mathrm{m}$. A $1800 \mathrm{~g} \mathrm{~mm}^{-1}$ grating was used to provide a spectral resolution of $0.5 \mathrm{~cm}^{-1}$. Diffuse reflectance spectroscopy (DRS) was analyzed with an ultraviolet-visible (UV-Vis) spectrophotometer (Thermo Scientific, Evolution 220) equipped with an integrating sphere detector.

\subsection{Selective Oxidation of $\mathrm{BzOH}$ Driven by Photothermal Effect}

The photothermal conversion was evaluated by continuously recording the temperature variation of the dispersion of colloidal nanoparticles under photo-illumination. In a typical measurement, $3 \mathrm{~mL}$ of nanoparticle dispersion was filled in a 4-mL glass vial. The vial was then wrapped with a layer of glass wool to minimize heat dissipation. Illuminating the nanoparticle dispersion with an LED light (Fiber-Lite Mi-LED series Illuminator, Dolan-Jenner) of $965 \mathrm{~mW}$ in power increased the temperature of the dispersion. Once the LED light was turned off, the temperature of the dispersion started to drop. The variation of temperature was continuously recorded with a ThermoWorks USB Reference Thermometer connected to a computer.

The selective oxidation of $\mathrm{BzOH}$ (Acros Organics) was performed in the presence of the as-synthesized $\mathrm{RuOOH} /$ $\mathrm{SiO}_{x}$ composite particles. Typically, $20 \mathrm{mg}$ of the $\mathrm{RuOOH} /$ $\mathrm{SiO}_{x}$ particles was dispersed in $3 \mathrm{~mL}$ of benzotrifluoride (BTF, Alfa Aesar) filled in a 4-mL glass vial that was sealed with a rubber septum. BTF was chosen as the solvent because of the good dispersity of the $\mathrm{RuOOH} / \mathrm{SiO}_{x}$ particles and the reasonable solubility of $\mathrm{BzOH}$ and $\mathrm{O}_{2}$. In a typical reaction, the dispersion was first purged with an $\mathrm{N}_{2} / \mathrm{O}_{2}$ gas containing varying concentrations of $\mathrm{O}_{2}$ in $\mathrm{N}_{2}$ at 1 atm for $15 \mathrm{~min}$. The atmosphere was then maintained above the dispersion, and the dispersion was illuminated with the LED light until the temperature became stable. To the photoilluminated dispersion was injected $46 \mu \mathrm{L}(0.444 \mathrm{mmol})$ of $\mathrm{BzOH}$, triggering the selective oxidation of $\mathrm{BzOH}$. The reactions in the dark were conducted by immersing the dispersion in a water bath that was used to tune the reaction temperature. The concentrations of $\mathrm{BzOH}$ and $\mathrm{BzAD}$ in reaction 
dispersion were analyzed by sampling aliquots of $0.2 \mathrm{~mL}$ dispersion. The aliquots were filtered with a $0.2-\mu \mathrm{m}$-poresize filter (SEOH) to remove the $\mathrm{RuOOH} / \mathrm{SiO}_{x}$ composite particles, and the filtrates were analyzed with a gas chromatography (GC) instrument (Agilent 7820A) equipped with an HP-5 column and a flame ionization detector (FID).

\section{Results and Discussion}

The $\mathrm{SiO}_{x}$ NSs synthesized through the controlled sol-gel process exhibit a uniform spherical geometry and uniform size with an average diameter of $443 \mathrm{~nm}$ (Fig. S1). Surface modification of the SiOx NSs with APTES does not change their geometrical and dimensional uniformity. The APTES-modified $\mathrm{SiO}_{x} \mathrm{NSs}$ expose amino groups (i.e., a type of Lewis base), which can grab protons from water $\left(\mathrm{H}_{2} \mathrm{O}\right)$ molecules to produce hydroxide ions $\left(\mathrm{OH}^{-}\right)$. In other words, concentrated hydroxide ions locally accumulate near the positively charged $-\mathrm{NH}_{3}{ }^{+}$groups through electrostatic interaction. As a result, adding $\mathrm{RuCl}_{3}$ to the aqueous dispersion of APTES-modified $\mathrm{SiO}_{x}$ NSs triggers the hydrolysis of $\mathrm{Ru}^{3+}$ primarily near the $-\mathrm{NH}_{3}{ }^{+}$groups, leading to the formation and condensation of $\mathrm{Ru}(\mathrm{OH})_{3}$ on the surfaces of the $\mathrm{SiO}_{x}$ NSs. The heterogeneous nucleation and growth of $\mathrm{Ru}(\mathrm{OH})_{3} \mathrm{NPs}$ on the $\mathrm{SiO}_{x} \mathrm{NSs}$ and the localized high concentration of $\mathrm{OH}^{-}$near the $\mathrm{SiO}_{x} \mathrm{NSs}$ accelerate the hydrolysis of $\mathrm{Ru}^{3+}$ to complete within $5 \mathrm{~s}$. By contrast, without surface modification of APTES, the hydrolysis of $\mathrm{Ru}^{3+}$ cannot rapidly and preferably occur on the surfaces of the $\mathrm{SiO}_{x} \mathrm{NSs}$ due to the low concentration of $\mathrm{OH}^{-}$throughout the reaction solution. Instead, homogeneous hydrolysis slowly proceeds in solution, resulting in the precipitation of black powders at the bottom of the reactor (Fig. S2).

Annealing the $\mathrm{Ru}(\mathrm{OH})_{3} / \mathrm{SiO}_{x}$ composite particles at elevated temperatures (e.g., $150{ }^{\circ} \mathrm{C}$ ) in air dehydrates the $\mathrm{Ru}(\mathrm{OH})_{3}$ nanoparticles, forming $\mathrm{RuOOH}$ ones by the reaction of Eq. 1:

$\mathrm{Ru}(\mathrm{OH})_{3} \stackrel{\Delta}{\longrightarrow} \mathrm{RuOOH}+\mathrm{H}_{2} \mathrm{O}$.

The dehydration treatment significantly increases the stability and wettability of the nanoparticles toward organic solvents of useful reactions. Figure 1a presents a typical SEM image of the $\mathrm{RuOOH} / \mathrm{SiO}_{x}$ composite particles, showing their intact monodispersity and overall spherical geometry. The TEM images of individual composite particles (Figs. 1b and $\mathrm{S} 1 \mathrm{~b}$ ) reveal that the RuOOH NPs with sizes of 2-3 nm are uniformly anchored to the surface of the $\mathrm{SiO}_{x} \mathrm{NSs}$. Ru $3 p$ XPS exhibits $3 p_{1 / 2}$ and $3 p_{3 / 2}$ features with binding energies of 485.3 and $463.5 \mathrm{eV}$ (Fig. 1c), respectively, confirming that the oxidation state of $\mathrm{Ru}$ is 3 [23, 24]. The consistent oxidation state of $\mathrm{Ru}$ in the precursor salt $\left(\mathrm{RuCl}_{3}\right)$ and the synthesized $\mathrm{RuOOH} / \mathrm{SiO}_{x}$ composite particles indicates the importance of mild thermal annealing that can stabilize the hydrolyzed nanoparticles but not over-oxidize the metal species. The stoichiometric ratio of $\mathrm{O}$ in $\mathrm{Ru}-\mathrm{O}-\mathrm{Ru}$ and $\mathrm{Ru}-\mathrm{OH}$ is determined from peak fitting of the O $1 s$ XPS signal (Fig. S3c), which shows the ratio close to 1:1. Therefore, the reasonable stoichiometric formula of the synthesized $\mathrm{Ru}$-containing NPs is RuOOH [25]. The Raman spectrum of the $\mathrm{RuOOH} / \mathrm{SiO}_{x}$ composite particles exhibits multiple peaks at 482,602 , and $790 \mathrm{~cm}^{-1}$, corresponding to the $E_{g}$, $A_{l g}$, and $B_{2 g}$ modes of Ru-O stretch vibrations, respectively $[26,27]$. The strong $\mathrm{Ru}-\mathrm{O}$ stretch vibrations indicate the formation of strong covalent bond between $\mathrm{Ru}$ and $\mathrm{O}$ in the small NPs (inset in Fig. 1b) loaded on the $\mathrm{SiO}_{x} \mathrm{NSs}$, which is consistent with the transformation of ionic-type $\mathrm{Ru}^{3+}$ / $\mathrm{OH}^{-}$bond in $\mathrm{Ru}(\mathrm{OH})_{3}$ NPs to covalent-type $\mathrm{Ru}(\mathrm{III})-\mathrm{O}$ bond in $\mathrm{RuOOH}$ nanoparticles through dehydrogenation reaction of Eq. 1. The absence of XPS signal near the $\mathrm{Cl} 2 p$ binding energy (Fig. S3b) confirms the complete removal of possibly adsorbed $\mathrm{Cl}^{-}$ions from the synthesized composite particles during the centrifuge/washing cycles.

The absorption-sensitive DRS spectrum of the assynthesized $\mathrm{RuOOH}$ powder in the absence of $\mathrm{SiO}_{x} \mathrm{NSs}$ exhibits a broad absorption peak around $430 \mathrm{~nm}$ that is attributed to the $p \rightarrow d$ interband transitions (blue curve, Fig. 2) [28]. The two minor sharp peaks at 686 and $825 \mathrm{~nm}$ correspond to the $\mathrm{Ru} t_{2 g}$ Drude-like intraband transitions. The pristine $\mathrm{SiO}_{x}$ NSs exhibit essentially no light absorption in the studied spectral region (black curve, Fig. 2). The $\mathrm{RuOOH} / \mathrm{SiO}_{x}$ composite particles shown in Fig. 1a, which are composed of ultrafine RuOOH NPs uniformly dispersed on the surfaces of the $\mathrm{SiO}_{x}$ NSs, exhibit a DRS spectrum (red curve, Fig. 2) significantly different from the sum of the spectra of both $\mathrm{RuOOH}$ powder and the $\mathrm{SiO}_{x}$ NSs. The light absorption intensity of the RuOOH/ $\mathrm{SiO}_{x}$ composite particles becomes much higher than that of the counterpart pure particles, indicating the role of the $\mathrm{SiO}_{x} \mathrm{NSs}$ in enhancing light absorption in the $\mathrm{RuOOH}$ NPs. Such enhancement intensifies the characteristic absorption peaks of the $\mathrm{RuOOH}$ nanoparticles although 

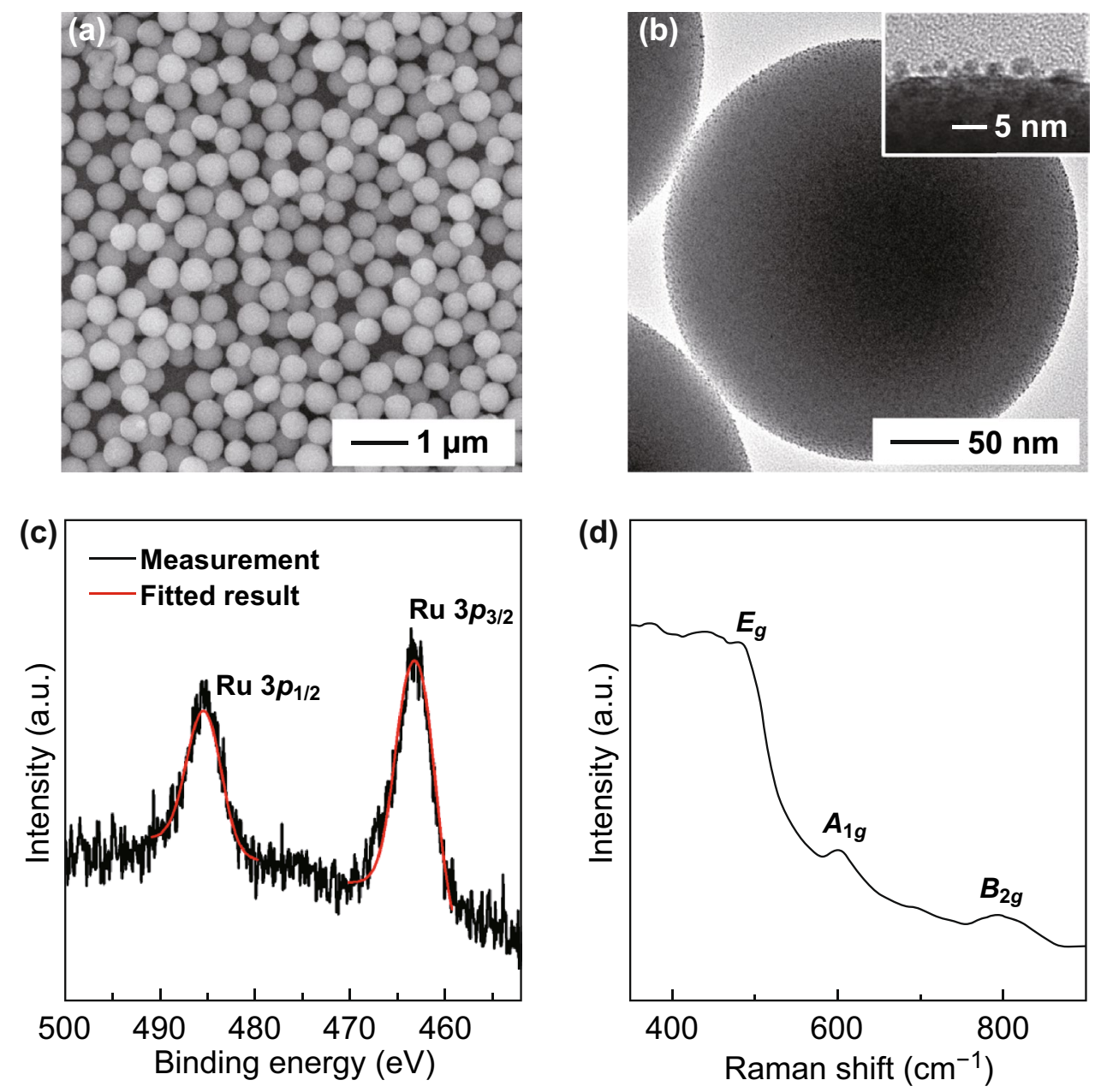

Fig. 1 a SEM image of $\mathrm{RuOOH} / \mathrm{SiO}_{x}$ composite particles. b TEM image of an individual $\mathrm{RuOOH} / \mathrm{SiO}_{x}$ composite particle, showing good dispersion of the RuOOH NPs uniformly distributed on the surface of the $\mathrm{SiO}_{x}$ NS. The inset of a blow-up TEM image highlights the well-defined and separated RuOOH NPs with diameters in the range of 2-3 nm. c Experimentally measured (black) and fitted (red) XPS spectrum of the Ru $3 p$ peaks for the $\mathrm{RuOOH} / \mathrm{SiO}_{x}$ composite particles. d Typical Raman spectrum of the $\mathrm{RuOOH} / \mathrm{SiO}_{x}$ composite particles. (Color figure online)

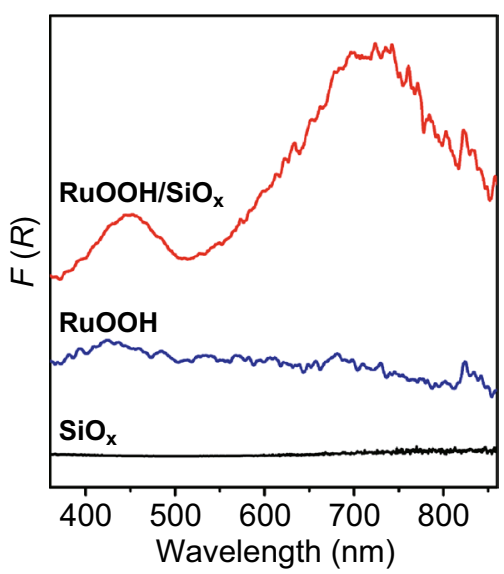

Fig. 2 DRS spectra of the synthesized $\mathrm{RuOOH} / \mathrm{SiO}_{x}$ composite particles (red curve), $\mathrm{RuOOH}$ powder (blue curve), and $\mathrm{SiO}_{x} \mathrm{NSs}$ (black curve). (Color figure online) their appearance may be concealed due to the overlap with other newly formed peaks. For example, the Ru $t_{2 g}$ intraband transition peaks are embedded in the new wide/ intense peak around $724 \mathrm{~nm}$. This additional strong peak is ascribed to the antenna effect of the $\mathrm{SiO}_{x}$ NSs on which strong surface light scattering resonances can occur to create significantly enhanced electric fields near the surfaces of the $\mathrm{SiO}_{x}$ NSs [18]. The ultrasmall RuOOH NPs dispersed on the $\mathrm{SiO}_{x} \mathrm{NSs}$ benefit from the locally intensified electric fields to enhance their light absorption power, showing strong absorption peaks correspondingly.

As discussed in Introduction, the weak light absorption in the highly dispersed small RuOOH NPs limits their use as an efficient photothermal catalyst for the selective 
aerobic oxidation of $\mathrm{BzOH}$. This challenge is overcome by loading the RuOOH NPs to the $\mathrm{SiO}_{x}$ NSs because of the antenna effect of the $\mathrm{SiO}_{x}$ NSs as highlighted in Fig. 2. A white LED lamp with an emission spectrum in the range of 400-700 nm (Fig. S4) has been used to evaluate the photothermal efficiency of the $\mathrm{RuOOH} / \mathrm{SiO}_{x}$ composite particles of Fig. 1a. The typical heating and cooling profile of a BTF dispersion of the $\mathrm{RuOOH} / \mathrm{SiO}_{x}$ composite particles is presented in Fig. S5a (red dots) when the LED lamp of $520 \mathrm{~mW}$ (i.e., power intensity of light interacting with the $\mathrm{RuOOH} / \mathrm{SiOx}$ particles in the test vial) is alternatively turned on and off. At the test condition, the dispersion of the $\mathrm{RuOOH} / \mathrm{SiO}_{x}$ composite particles shows a temperature increase of $23.1{ }^{\circ} \mathrm{C}$ at equilibrium under the continuous light illumination, whereas the dispersion of the same amount of pristine $\mathrm{RuOOH}$ powder (in the absence of $\mathrm{SiO}_{x} \mathrm{NSs}$ ) shows a temperature increase of only $9{ }^{\circ} \mathrm{C}$ (Fig. S5a, red dots versus blue dots). The $\mathrm{SiO}_{x} \mathrm{NSs}$ do not show an observable photothermal effect (Fig. S5a, black dots). The comparison confirms that both the small size of nanoparticles of a light-absorbing material and the antenna effect of the $\mathrm{SiO}_{x}$ NSs are beneficial to high photothermal efficiency.

Because of the importance of aerobic selective oxidation of $\mathrm{BzOH}$ to $\mathrm{BzAD}$ and the catalytic activity of the small RuOOH nanoparticles, the selective oxidation of $\mathrm{BzOH}$ to $\mathrm{BzAD}$ has been chosen to evaluate the efficiency of the $\mathrm{RuOOH} / \mathrm{SiO}_{x}$ composite particles as a new class of photothermal catalyst. In a reaction solution containing a given amount of $\mathrm{RuOOH} / \mathrm{SiO}_{x}$ particles, the equilibrium temperature reached under the illumination of an LED lamp can be tuned by varying the light power density. The solution temperature $(T)$ exhibits a linear dependence, $T / \mathrm{K}=294.35+45.79 P / \mathrm{W}$, on the effective light power density $\left(P_{\text {eff }}\right)$ in the range of $161-520 \mathrm{~mW}$ (Fig. 3, blue triangles). The corresponding photothermal energy conversion efficiency of the absorbed light in the $\mathrm{RuOOH}$ nanoparticles, $\eta_{\text {abs }}$, is close to the unity (or $\sim 100 \%$ ), and the apparent total photo-to-thermal efficiency with respect to the power of light interacting with the $\mathrm{RuOOH} / \mathrm{SiOx}$ composite particles $\left(\eta_{\text {total }}\right)$ is $92.47 \%$ (Figs. S5 and S6).

The reaction rate of $\mathrm{BzOH}-$ to-BzAD conversion in the presence of the $\mathrm{RuOOH} / \mathrm{SiO}_{x}$ composite particles is $0.36 \mu \mathrm{M} \mathrm{s}^{-1}$ (Fig. S7, blue dots), corresponding to a turnover frequency (TOF) of $5.2 \times 10^{-4} \mathrm{~s}^{-1}$ normalized against the number of $\mathrm{Ru}$ atoms, at $24{ }^{\circ} \mathrm{C}$ in the dark under the

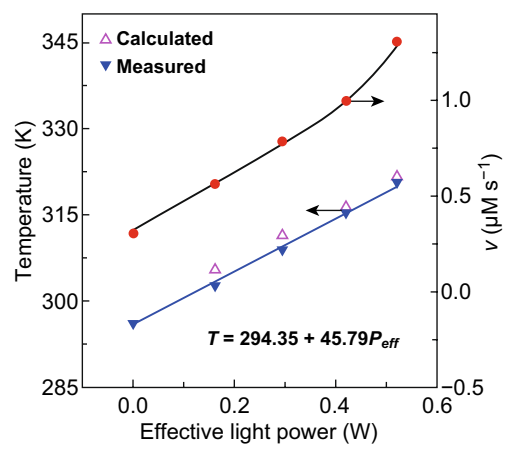

Fig. 3 Photothermally induced increase in (triangles) temperature of the reaction solution and (solid red dots) reaction rate in the presence of the $\mathrm{RuOOH} / \mathrm{SiO}_{x}$ composite particles as a photothermal catalyst. Blue triangles indicate the temperature measured by the thermocouple probe under the illumination of different effective light powers $\left(P_{\text {eff }}\right)$, showing a linear dependence. Violet triangles correspond to the reaction temperatures calculated from the measured reaction rates (solid red dots) according to the Arrhenius equation. (Color figure online)

atmosphere of $1 \mathrm{~atm} \mathrm{O}_{2}$. Illuminating the reaction solution accelerates the oxidation reaction along with the increase in temperature (Fig. 3, red dots). For instance, the reaction rate increases to $1.30 \mu \mathrm{M} \mathrm{s}^{-1}$ (or TOF of $1.89 \times 10^{-3} \mathrm{~s}^{-1}$ ) under the illumination of effective power of $520 \mathrm{~mW}$ (Fig. S7, red dots), highlighting that the photothermally induced temperature increase is indeed capable of accelerating the oxidation of $\mathrm{BzOH}$. To further confirm that the reaction rate is solely improved by the photothermal effect, the oxidation of $\mathrm{BzOH}$ reaction is performed at a constant temperature of $35^{\circ} \mathrm{C}$ by immersing the reaction vial in a large-volume water bath, while light illumination is alternatively turned on and off. Regardless of the photo-illumination condition, the reaction exhibits a constant reaction rate that is determined from the constant slope of the time-dependent yield of BzAD (Fig. S8). The unchanged reaction rate in the course of the entire oxidation reaction excludes the involvement of hot-electron chemistry that usually significantly boosts reaction kinetics. In contrast, the control experiments in the absence of $\mathrm{RuOOH} / \mathrm{SiO}_{x}$ composite particles or in the presence of only $\mathrm{SiO}_{x}$ NSs do not show the detectable formation of BzAD (Table S1). Moreover, both the pristine $\mathrm{RuOOH}$ powder of large particles (Fig. S9a) and the agglomerated RuOOH NPs of large sizes on $\mathrm{SiO}_{x} \mathrm{NSs}$ (Fig. S9b) cannot catalyze the oxidation of $\mathrm{BzOH}$ even though the temperature of the reaction solution elevates under photo-illumination. Comparison with the control experiments indicates that the small size of 
the RuOOH NPs is crucial to achieving the high catalytic activity, which can be further enhanced by the photothermal effect. The longtime stability has been evaluated using the $\mathrm{RuOOH} / \mathrm{SiO}_{x}$ hybrid particles of Fig. 1 as the photothermal catalyst. No obvious decrease in reaction rate is observed even after the reaction lasts $24 \mathrm{~h}$ under photo-illumination (Fig. S10). The TEM images of the $\mathrm{RuOOH} / \mathrm{SiO}_{x}$ composite particles after the $24-\mathrm{h}$ reaction indicate that the composite particles, in particular the small RuOOH NPs, are intact (inset, Fig. S10). The retaining of both the photothermal catalytic activity and the structural geometry/integrity of the $\mathrm{RuOOH} / \mathrm{SiO}_{x}$ composite particles after a longtime reaction highlights the stability of the $\mathrm{RuOOH} / \mathrm{SiO}_{x}$ catalyst.

The activation energy of the selective oxidation of $\mathrm{BzOH}$ on the RuOOH NPs is determined as $41.75 \mathrm{~kJ} \mathrm{~mol}^{-1}$ from the dependence of reaction rate in the dark on the solution temperature according to the Arrhenius equation (Fig. S11). Inserting these reaction rates measured under light illumination of varying power densities into the Arrhenius equation, we can estimate the reaction temperatures (Fig. 3, open violet triangles) necessary to support the observed reaction rates. The good agreement between the estimated temperatures and the solution temperatures directly measured with the thermocouple (open triangles versus solid triangles, Fig. 3) indicates that the acceleration of the oxidation reaction in the presence of $\mathrm{RuOOH} / \mathrm{SiO}_{x}$ composite particles under photo-illumination is primarily ascribed to the photothermal effect.

The selective oxidation of $\mathrm{BzOH}$ to $\mathrm{BzAD}$ in the presence of $\mathrm{RuOOH} / \mathrm{SiO}_{x}$ composite particles strongly depends on the partial pressure of $\mathrm{O}_{2}$ (i.e., the reaction rate increases with the partial pressure of $\mathrm{O}_{2}$ ). The observation is significantly different from the previously reported hydrated $\mathrm{RuO}_{2}$ and $\mathrm{Ru}(\mathrm{OH})_{3}$ catalysts on which the rate-determining step (RDS) of oxidation reactions involves the elimination of $\beta-\mathrm{H}$ of $\mathrm{BzOH}$ and is independent of the partial pressure of $\mathrm{O}_{2}$ [12-15]. The difference indicates a different reaction mechanism on the ultrasmall RuOOH NPs, where oxygen involves in the RDS of oxidizing $\mathrm{BzOH}$. The freestanding molecular oxygen, $\mathrm{O}_{2}$, is inert at mild temperature toward the elimination of $\beta-\mathrm{H}$ of $\mathrm{BzOH}$ to drive $\mathrm{BzOH}$-to- $\mathrm{BzAD}$ conversion $[29,30]$. The coordination number of $\mathrm{Ru}$ in the $\mathrm{RuOOH}$ solids is 3 and most likely to decrease to 2 on the surface [31-33]. The low coordination number of surface Ru(III) in the small $\mathrm{RuOOH}$ NPs leads to the easy adsorption of $\mathrm{O}_{2}$ by forming coordination bonds between $\mathrm{O}_{2}$ and $\mathrm{Ru}$. $\mathrm{Ru}(\mathrm{III})$ possesses the ability to partially donate one electron to the antibonding orbital of the flat-on/end-on adsorbed $\mathrm{O}_{2}$, weakening the oxygen-oxygen double bonds to increase the reactivity of adsorbed $\mathrm{O}_{2}$. The activated $\mathrm{O}_{2}$ reacts with $\beta-\mathrm{H}$ of $\mathrm{BzOH}$ to form BzAD. Such reaction mechanism involving the activation of adsorbed $\mathrm{O}_{2}$ is supported by fitting the reaction kinetics using the Langmuir-Hinshelwood $(\mathrm{L}-\mathrm{H})$ mechanism (Eq. 2) where both $\mathrm{BzOH}$ and $\mathrm{O}_{2}$ are adsorbed on RuOOH NPs [34, 35]:

$v=\frac{\gamma K_{1} K_{2}[\mathrm{BzOH}]\left[\mathrm{O}_{2}\right]_{\mathrm{sol}}^{n}}{\left[1+K_{1}[\mathrm{BzOH}]+K_{2}\left[\mathrm{O}_{2}\right]_{\mathrm{sol}}^{n}\right]^{2}}$,

where $\gamma$ is the rate coefficient, $K_{1}$ and $K_{2}$ are the adsorption equilibrium constants defined by the ratio of rates of surface adsorption and desorption for $\mathrm{BzOH}$ and $\mathrm{O}_{2}$, respectively, which depend on temperature and the surface chemistry of catalysts. The value of $n$ can be taken as 1 and 0.5 for associative and dissociative adsorption of $\mathrm{O}_{2}$, respectively. The concentration of $\mathrm{O}_{2}$ dissolved in the reaction solution is proportional to its partial pressure, $p_{\mathrm{O}_{2}}$, following the Henry's law (Eq. 3):

$\left[\mathrm{O}_{2}\right]_{\mathrm{sol}}=\frac{1}{K_{\mathrm{H}}} p_{\mathrm{O}_{2}}$.

$K_{\mathrm{H}}$ is a temperature $(T)$-dependent constant determined by Eq. 4:

$K_{\mathrm{H}}=K_{\mathrm{H}}^{\theta} \exp \left[-\alpha\left(\frac{1}{T}-\frac{1}{T^{\theta}}\right)\right]$,

where $K_{\mathrm{H}}^{\theta}$ and $T^{\ominus}$ are the Henry's constant and temperature at the standard condition and $\alpha$ is a constant.

When molecular oxygen is associatively adsorbed on the RuOOH NPs, Eq. 2 can be simplified to Eq. 5:

$\sqrt{\frac{p_{\mathrm{O}_{2}}}{v}}=\frac{b}{\sqrt{a}}+\frac{c}{\sqrt{a}} p_{\mathrm{O}_{2}}$,

where $a=\gamma K_{1} K_{2}[\mathrm{BzOH}], b=1+K_{1}[\mathrm{BzOH}]$, and $c=K_{2} / K_{\mathrm{H}}$. At a given temperature and a high concentration of $\mathrm{BzOH}$, $a, b$, and $c$ are considered as constants at the early reaction stage when the conversion of $\mathrm{BzOH}$ is low (e.g., $<5 \%$ ). Similarly, for a reaction involving the dissociative adsorption of $\mathrm{O}_{2}$, Eq. 2 becomes Eq. 6:

$\frac{\sqrt[4]{p_{\mathrm{O}_{2}}}}{\sqrt{v}}=\frac{b}{\sqrt{a}}+\frac{c}{\sqrt{a}} \sqrt{p_{\mathrm{O}_{2}}}$. 


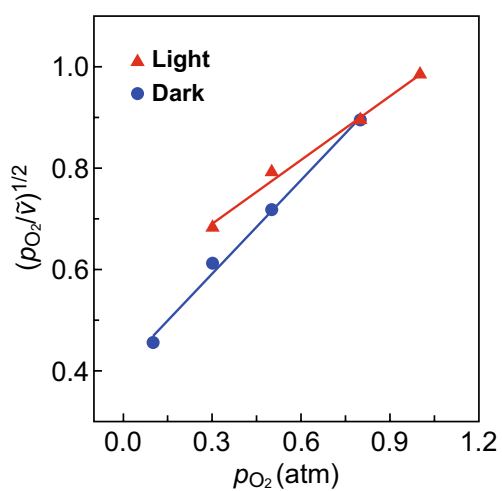

Fig. 4 Dependence of reaction rate with a partial pressure of $\mathrm{O}_{2}$ $\left(p_{\mathrm{O}_{2}}\right)$ in the atmosphere, tested under photo-illumination of an effective light power of $520 \mathrm{~mW}$ (red dots) and dark condition (blue dots). The results are plotted according to the linear form of the LangmuirHinshelwood model in which oxygen molecules are associatively adsorbed on the catalyst surface. $\tilde{v}$ represents the reaction rates normalized against the corresponding reaction rate under $0.8 \mathrm{~atm}$. (Color figure online)

The fitting results shown in Figs. 4 and S13 indicate that oxygen is associatively adsorbed on the ultrasmall $\mathrm{RuOOH}$ NPs. The consistent linear dependence of $\sqrt{\frac{p_{\mathrm{O}_{2}}}{v}}$ on the partial pressure of $\mathrm{O}_{2}$ in the dark and under photo-illumination (Fig. 4 red dots versus blue dots) indicates that photoexcitation of the RuOOH NPs does not change the reaction mechanism. The variation of slope and intercept of the linear fitting for the reaction in the dark and under light is ascribed to the increased temperature under light illumination, which influences the constants, $a, b$, and $c$, in Eq. 5. The dependence of the reaction rate on the partial pressure of $\mathrm{O}_{2}$ offers a new strategy to control the selective oxidation of $\mathrm{BzOH}$ by simply tuning the reaction atmosphere in the presence of the supported ultrasmall RuOOH NPs.

\section{Conclusion}

Ultrafine RuOOH NPs with sizes of $2-3 \mathrm{~nm}$ have been successfully synthesized on the surfaces of APTES-modified $\mathrm{SiO}_{x} \mathrm{NSs}$ through controlled hydrolysis of $\mathrm{Ru}^{3+}$ ions followed by mild thermal annealing at elevated temperatures. The ultrafine RuOOH NPs are uniformly dispersed on the surfaces of the $\mathrm{SiO}_{x}$ NSs, exhibiting a high dispersion to expose the large surface area and minimize the usage of $\mathrm{Ru}$ as a catalyst. The ultrasmall RuOOH NPs become capable of activating molecular oxygen adsorbed on their surfaces to drive the selective aerobic oxidation of $\mathrm{BzOH}$ under ambient conditions, contrast to Ru-containing nanoparticles with larger sizes. The $\mathrm{SiO}_{x} \mathrm{NSs}$ in the $\mathrm{RuOOH} / \mathrm{SiO}_{x}$ composite particles play two essential functions to benefit the composite particles to be an efficient photothermal catalyst. First, the $\mathrm{SiO}_{x}$ NSs serve as support to maintain the high dispersion of the ultrasmall RuOOH NPs and good colloidal stability even under reaction conditions as shown in this work. Second, the intense surface light scattering resonances on the $\mathrm{SiO}_{x}$ NSs significantly increase the light absorption power of the RuOOH NPs and thus their photothermal conversion efficiency even with the reduced amount of Ru. Forming the $\mathrm{RuOOH} / \mathrm{SiO}_{x}$ composite particles, for the first time, enables photothermal catalysis of selective aerobic oxidation of $\mathrm{BzOH}$ to BzAD with high energy efficiency. The materials' design and synthesis described in this work open a promising avenue to explore the unique properties of functional NPs enabled by the ultrasmall sizes.

Acknowledgements This work was supported by the start-up from Temple University. Partial characterizations were performed with the use of TMI (Temple Materials Institute) facilities. The authors thank Yu Wang and Dr. Robert J. Levis for their help on Raman spectroscopy.

Open Access This article is licensed under a Creative Commons Attribution 4.0 International License, which permits use, sharing, adaptation, distribution and reproduction in any medium or format, as long as you give appropriate credit to the original author(s) and the source, provide a link to the Creative Commons licence, and indicate if changes were made. The images or other third party material in this article are included in the article's Creative Commons licence, unless indicated otherwise in a credit line to the material. If material is not included in the article's Creative Commons licence and your intended use is not permitted by statutory regulation or exceeds the permitted use, you will need to obtain permission directly from the copyright holder. To view a copy of this licence, visit http://creativecommons.org/licenses/by/4.0/.

Electronic supplementary material The online version of this article (https://doi.org/10.1007/s40820-020-0375-9) contains supplementary material, which is available to authorized users.

\section{References}

1. T. Mallat, A. Baiker, Oxidation of alcohols with molecular oxygen on solid catalysts. Chem. Rev. 104, 3037-3058 (2004). https://doi.org/10.1021/cr0200116

2. U.R. Pillai, E. Sahle-Demessie, Selective oxidation of alcohols by molecular oxygen over a $\mathrm{Pd} / \mathrm{MgO}$ catalyst in the absence 
of any additives. Green Chem. 6, 161-165 (2004). https://doi. org/10.1039/B316414B

3. P.A. Shapley, N. Zhang, J.L. Allen, D.H. Pool, H.-C. Liang, Selective alcohol oxidation with molecular oxygen catalyzed by $\mathrm{Os}-\mathrm{Cr}$ and $\mathrm{Ru}-\mathrm{Cr}$ complexes. J. Am. Chem. Soc. 122, 1079-1091 (2000). https://doi.org/10.1021/ja982171y

4. T. Yasu-eda, S. Kitamura, N.-O. Ikenaga, T. Miyake, T. Suzuki, Selective oxidation of alcohols with molecular oxygen over $\mathrm{Ru} / \mathrm{CaO}-\mathrm{ZrO}_{2}$ catalyst. J. Mol. Catal. A 323, 7-15 (2010). https://doi.org/10.1016/j.molcata.2010.03.018

5. F. Li, Q. Zhang, Y. Wang, Size dependence in solvent-free aerobic oxidation of alcohols catalyzed by zeolite-supported palladium nanoparticles. Appl. Catal. A 334, 217-226 (2008). https://doi.org/10.1016/j.apcata.2007.10.008

6. J.A. Mueller, C.P. Goller, M.S. Sigman, Elucidating the significance of $\beta$-hydride elimination and the dynamic role of acid/base chemistry in a palladium-catalyzed aerobic oxidation of alcohols. J. Am. Chem. Soc. 126, 9724-9734 (2004). https://doi.org/10.1021/ja047794s

7. J. Xu, J.-K. Shang, Y. Chen, Y. Wang, Y.-X. Li, Palladium nanoparticles supported on mesoporous carbon nitride for efficiently selective oxidation of benzyl alcohol with molecular oxygen. Appl. Catal. A 542, 380-388 (2017). https://doi. org/10.1016/j.apcata.2017.05.036

8. J.-B. Chang, C.-H. Liu, J. Liu, Y.-Y. Zhou, X. Gao, S.-D. Wang, Green-chemistry compatible approach to $\mathrm{TiO}_{2}$-supported PdAu bimetallic nanoparticles for solventfree 1-phenylethanol oxidation under mild conditions. NanoMicro Lett. 7, 307-315 (2015). https://doi.org/10.1007/s4082 0-015-0044-6

9. A.A.S. Oliveira, D.S. Costa, I.F. Teixeira, L.A. Parreira, L. Menini, E.V. Gusevskaya, F.C.C. Moura, Red mud based gold catalysts in the oxidation of benzyl alcohol with molecular oxygen. Catal. Today 289, 89-95 (2017). https://doi. org/10.1016/j.cattod.2016.10.028

10. S. Cao, F. Tao, Y. Tang, Y. Li, J. Yu, Size- and shape-dependent catalytic performances of oxidation and reduction reactions on nanocatalysts. Chem. Soc. Rev. 45, 4747-4765 (2016). https://doi.org/10.1039/C6CS00094K

11. F. Wang, Y. Xu, K. Zhao, D. He, Preparation of palladium supported on ferric oxide nano-catalysts for carbon monoxide oxidation in low temperature. Nano-Micro Lett. 6, 233-241 (2014). https://doi.org/10.1007/BF03353787

12. F. Li, J. Chen, Q. Zhang, Y. Wang, Hydrous ruthenium oxide supported on $\mathrm{Co}_{3} \mathrm{O}_{4}$ as efficient catalyst for aerobic oxidation of amines. Green Chem. 10, 553-562 (2008). https://doi. org/10.1039/B715627H

13. K. Yamaguchi, N. Mizuno, Supported ruthenium catalyst for the heterogeneous oxidation of alcohols with molecular oxygen. Angew. Chem. Int. Ed. 114(23), 4720-4724 (2002). https://doi.org/10.1002/15213773(20021202)41:23\%3c453 8:AIDANIE4538\%3e3.0.CO;2-6

14. J.B. Brazier, K. Hellgardt, K.K. Hii, Catalysis in flow: $\mathrm{O}_{2}$ effect on the catalytic activity of $\mathrm{Ru}(\mathrm{OH})_{x} / \gamma-\mathrm{Al}_{2} \mathrm{O}_{3}$ during the aerobic oxidation of an alcohol. React. Chem. Eng. 2, 60-67 (2017). https://doi.org/10.1039/C6RE00208K
15. K. Yamaguchi, T. Koike, M. Kotani, M. Matsushita, S. Shinachi, N. Mizuno, Synthetic scope and mechanistic studies of $\mathrm{Ru}(\mathrm{OH})_{x} / \mathrm{Al}_{2} \mathrm{O}_{3}$-catalyzed heterogeneous hydrogen-transfer reactions. Chem. Eur. J. 11, 6574-6582 (2005). https://doi. org/10.1002/chem.200500539

16. S. Mei, Z. Kochovski, R. Roa, S. Gu, X. Xu, H. Yu, J. Dzubiella, M. Ballauff, Y. Lu, Enhanced catalytic activity of gold@polydopamine nanoreactors with multi-compartment structure under NIR irradiation. Nano-Micro Lett. 11, 83 (2019). https://doi.org/10.1007/s40820-019-0314-9

17. B.N. Ganguly, B. Maity, T.K. Maity, J. Manna, M. Roy et al., L-cysteine-conjugated ruthenium hydrous oxide nanomaterials with anticancer active application. Langmuir 34, 1447-1456 (2018). https://doi.org/10.1021/acs.langmuir.7b01408

18. X. Dai, K.D. Rasamani, G. Hall, R. Makrypodi, Y. Sun, Geometric symmetry of dielectric antenna influencing light absorption in quantum-sized metal nanocrystals: a comparative study. Front. Chem. 6, 494 (2018). https://doi.org/10.3389/ fchem.2018.00494

19. X. Dai, K.D. Rasamani, S. Wu, Y. Sun, Enabling selective aerobic oxidation of alcohols to aldehydes by hot electrons in quantum-sized Rh nanocubes. Mater. Today Energy 10, 15-22 (2018). https://doi.org/10.1016/j.mtener.2018.08.003

20. X. Dai, Q. Wei, T. Duong, Y. Sun, Selective transfer coupling of nitrobenzene to azoxybenzene on $\mathrm{Rh}$ nanoparticle catalyst promoted by photoexcited hot electrons. ChemNanoMat 5, 1000-1007 (2019). https://doi.org/10.1002/ cnma.201900182

21. N. Zhang, C. Han, Y.-J. Xu, J.J. Foley IV, D. Zhang, J. Codrington, S.K. Gray, Y. Sun, Near-field dielectric scattering promotes optical absorption by platinum nanoparticles. Nat. Photon. 10, 473-482 (2016). https://doi.org/10.1038/nphot on.2016.76

22. L. Zhou, D.F. Swearer, C. Zhang, H. Robatjazi, H. Zhao et al., Quantifying hot carrier and thermal contributions in plasmonic photocatalysis. Science 362, 69-72 (2018). https ://doi.org/10.1126/science.aat6967

23. NIST X-ray Photoelectron Spectroscopy Database, NIST Standard Reference Database Number 20, National Institute of Standards and Technology, Gaithersburg MD, 20899. DOI: 10.18434/T4T88K

24. D.J. Morgan, Resolving ruthenium: XPS studies of common ruthenium materials. Surf. Interface Anal. 47, 1072-1079 (2015). DOI: 10.1002/sia.5852

25. W. Wang, S. Guo, I. Lee, K. Ahmed, J. Zhong et al., Hydrous ruthenium oxide nanoparticles anchored to graphene and carbon nanotube hybrid foam for supercapacitors. Sci. Rep. 4, 4452 (2014). https://doi.org/10.1038/srep04452

26. J. Jin, X. Yan, S. Xu, S. Liu, J. Hong, F. Huang, Y. Dai, C. Jin, J. Fan, Exploration of the interaction of $\mathrm{RuO}_{2}-\mathrm{Au}$ composite nanoparticles formed by one-step synthesis within the mesopores. Chem. Mater. 25, 3921-3927 (2013). https://doi. org/10.1021/cm4025233

27. L.-J. Meng, M.P. dos Santos, A study of residual stress on rf reactively sputtered $\mathrm{RuO}_{2}$ thin films. Thin Solid Films 375, 
29-32 (2000). https://doi.org/10.1016/S0040-6090(00)01174 $-3$

28. J.S. de Almeida, R. Ahuja, Electronic and optical properties of $\mathrm{RuO}_{2}$ and $\mathrm{IrO}_{2}$. Phys. Rev. B 73, 165102 (2006). https://doi. org/10.1103/PhysRevB.73.165102

29. X. Deng, B.K. Min, A. Guloy, C.M. Friend, Enhancement of $\mathrm{O} 2$ dissociation on $\mathrm{Au}(111)$ by adsorbed oxygen: implications for oxidation catalysis. J. Am. Chem. Soc. 127, 9267-9270 (2005). https://doi.org/10.1021/ja050144j

30. P. Légaré, L. Hilaire, M. Sotto, G. Maire, Interaction of oxygen with Au surfaces: A LEED, AES and ELS study. Surf. Sci. 91, 175-186 (1980). https://doi.org/10.1016/0039-6028(80)90078 $-3$

31. X. Han, X. Han, L. Sun, S. Gao, L. Li, Q. Kuang, Z. Xie, C. Wang, Synthesis of trapezohedral indium oxide nanoparticles with high-index 211 facets and high gas sensing activity. Chem. Commun. 51, 9612-9615 (2015). https://doi. org/10.1039/c5cc02029h

32. S. Xiong, W. Qi, Y. Cheng, B. Huang, M. Wang, Y. Li, Modeling size effects on the surface free energy of metallic nanoparticles and nanocavities. Phys. Chem. Chem. Phys. 13, 10648-10651 (2011). https://doi.org/10.1039/C0CP02102D

33. W. Qi, B. Huang, M. Wang, Structure of unsupported small palladium nanoparticles. Nanoscale Res. Lett. 4, 269 (2009). https://doi.org/10.1007/s11671-008-9236-z

34. M. Ilyas, M. Sadiq, Liquid-phase aerobic oxidation of benzyl alcohol catalyzed by $\mathrm{Pt} / \mathrm{ZrO}_{2}$. Chem. Eng. Technol. 30, 1391-1397 (2007). https://doi.org/10.1002/ceat.200700072

35. V.D. Makwana, Y.-C. Son, A.R. Howell, S.L. Suib, The role of lattice oxygen in selective benzyl alcohol oxidation using OMS-2 catalyst: a kinetic and isotope-labeling study. J. Catal. 210, 46-52 (2002). https://doi.org/10.1006/jcat.2002.3680 